\title{
Synergies Between Human and Animal Health Syndromic Surveillance: Triple-S Outputs
}

\author{
Céline Dupuy*1, Jean-Baptiste Perrin ${ }^{1}$, Anne Bronner ${ }^{1}$, Didier Calavas ${ }^{1}$, Pascal Hendrikx ${ }^{2}$ \\ and Anne Fouillet ${ }^{3}$
}

${ }^{1}$ French Agency for food, environmental and occupational health safety (Anses), Lyon, France; ${ }^{2}$ French Agency for food environmental and occupational health safety (Anses), Maisons-Alfort, France; ${ }^{3}$ French institute for public health surveillance (InVS), Saint-Maurice, France

\section{Objective}

The objective of this study, based on the Triple-S project outputs, was to present the existing synergies between human and animal health syndromic surveillance (SyS) systems in Europe and a proposal to enhance this kind of collaboration.

\section{Introduction}

The Triple-S project (Syndromic Surveillance Systems in Europe, www.syndromicsurveillance.eu), co-financed by the European Commission and involving twenty four organizations from fourteen countries was launched in September 2010 with the following objectives 1) performing an inventory of existing or planned SyS systems in Europe both in animal and public health, 2) building a network of experts involved in SyS 3) producing guidelines to implement SyS systems, 4) developing synergies between human and animal health SyS systems. The project is based on a cooperation between human and animal health experts, as supported by the One Health initiative [1].

\section{Methods}

A network of European experts involved in SyS was identified through the Triple-S inventory of SyS systems. A meeting of human health experts was organized back to back with a similar meeting with animal health experts in Paris, September 12-14, 2011. A joint session human/animal health allowed experts to discuss the interest of synergies between both sides. The objectives were to 1) encourage experience and knowledge transfer, 2) discuss what and how information should be shared between both sides to improve respective performances.

\section{Results}

The results of the inventory of veterinary SyS systems showed that $40 \%$ of identified systems already shared or had planned to share information with human health sector. For these systems the collaboration between human and animal health sectors consisted in meetings on a regular basis to discuss the surveillance results.

Discussions during the Triple-S meeting highlighted two reasons for enhancing synergies between both sides. First human health and animal health epidemiologists face common statistical and epidemiological issues when dealing with SyS, i.e. use of data collected for other purpose than surveillance; standardization of clinical observations; syndrome definition; anomaly detection; interpretation of unspecific signals; response to alerts. Both sides have thus interest in sharing their experiences and knowledge to improve their respective systems.
Second, systems on both sides have similar objectives and target health events potentially threatening both animal and human populations: zoonoses, extreme weather events, environmental / food contamination, bioterrorist attack... For those events, animal population can play the role of sentinel for human population. Regular information flow between human and animal SyS could thus enhance the timeliness and sensitivity of SyS systems for detecting unexpected health events. Moreover, sharing information could help animal and human health experts to interpret and confirm unspecific signals, and confirm the impact of common health threats.

All participants of the meeting agreed on the idea to routinely share outputs of the systems but were sceptical about sharing raw data to perform global analysis.

\section{Conclusions}

Each aspect of the Triple-S project includes both human and animal health and will thus contribute to build natural collaboration between both sides. Such a project has demonstrated that scientific community is more and more willing to collaborate beyond the boundaries of these two health fields.

Synergies between human and animal health seem as necessary for syndromic surveillance as it is for traditional surveillance, if not more. They seem especially important for the detection of emerging zoonotic threats but not only. Sharing surveillance outputs from both sides would be the first step of collaboration but deeper synergy, e.g. sharing data and analyse them globally, could also be considered. Triple-S guidelines for implementation of SyS systems in Europe will take into account and promote synergies between human and animal health.

\section{Keywords}

syndromic surveillance; synergy; early warning

\section{Acknowledgments}

The authors thank all participants to the Triple-S project activities.

\section{References}

1.Zinsstag, J., et al., Potential of cooperation between human and animal health to strengthen health systems. The Lancet, 2005. 366(9503): $\mathrm{p}$ 2142-2145.

\section{*Céline Dupuy}

E-mail: celine.dupuy@anses.fr 